\title{
The Short Physical Performance Battery is a
} discriminative tool for identifying patients with COPD at risk of disability

This article was published in the following Dove Press journal:

International Journal of COPD

3 December 2015

Number of times this article has been viewed

Roberto Bernabeu-Mora ${ }^{1,2}$

Françesc Medina-Mirapeix ${ }^{2}$

Eduardo Llamazares-Herrán ${ }^{3}$

Gloria García-Guillamón ${ }^{2}$

Luz María Giménez-Giménez ${ }^{2}$

Juan Miguel Sánchez-Nieto ${ }^{1,4}$

'Division of Pneumology, Hospital Morales Meseguer, ${ }^{2}$ Department of Physical Therapy, University of Murcia, Murcia, ${ }^{3}$ Department of Physical Therapy, Alcala University, Alcala de Henares, ${ }^{4}$ Department of Intern Medical, University of Murcia, Murcia, Spain

Correspondence: Roberto BernabeuMora

Division of Pneumology, Hospital General Universitario Morales Meseguer, Avda Marqués de los Velez s/n, 30008 Murcia, Spain

Tel +34968360900

Fax +34968360994

Email rbernabeumora@hotmail.com
Background: Limited mobility is a risk factor for developing chronic obstructive pulmonary disease (COPD)-related disabilities. Little is known about the validity of the Short Physical Performance Battery (SPPB) for identifying mobility limitations in patients with COPD.

Objective: To determine the clinical validity of the SPPB summary score and its three components (standing balance, 4-meter gait speed, and five-repetition sit-to-stand) for identifying mobility limitations in patients with COPD.

Methods: This cross-sectional study included 137 patients with COPD, recruited from a hospital in Spain. Muscle strength tests and SPPB were measured; then, patients were surveyed for selfreported mobility limitations. The validity of SPPB scores was analyzed by developing receiver operating characteristic curves to analyze the sensitivity and specificity for identifying patients with mobility limitations; by examining group differences in SPPB scores across categories of mobility activities; and by correlating SPPB scores to strength tests.

Results: Only the SPPB summary score and the five-repetition sit-to-stand components showed good discriminative capabilities; both showed areas under the receiver operating characteristic curves greater than 0.7. Patients with limitations had significantly lower SPPB scores than patients without limitations in nine different mobility activities. SPPB scores were moderately correlated with the quadriceps test $(r>0.40)$, and less correlated with the handgrip test $(r<0.30)$, which reinforced convergent and divergent validities. A SPPB summary score cutoff of 10 provided the best accuracy for identifying mobility limitations.

Conclusion: This study provided evidence for the validity of the SPPB summary score and the fiverepetition sit-to-stand test for assessing mobility in patients with COPD. These tests also showed potential as a screening test for identifying patients with COPD that have mobility limitations.

Keywords: disability, COPD, mobility, SPPB, five-repetition sit-to-stand, 4-meter gait speed

\section{Introduction}

Prior research has indicated that chronic obstructive pulmonary disease (COPD) is associated with reduced mobility in performing activities necessary for living independently. ${ }^{1}$ Mobility refers to actions related to maintaining or changing body positions (eg, stooping, crouching, or kneeling,), carrying or moving objects (eg, lifting or carrying light or heavy items), or walking and moving (eg, walking up stairs, walking around the neighborhood), as suggested by the International Classification of Functioning, Disability, and Health (ICF). ${ }^{2}$ Based on this classification, mobility limitation can be defined as a difficulty encountered by an individual in executing some of those tasks or actions.

The development of limitations in mobility is an important event in the course of COPD, because these limitations negatively affect independence. Moreover, 
limitations in mobility represent a potential risk factor for the development of new disabilities in other kinds of activities (domestic life, self-care, social and recreational activities, etc). ${ }^{3}$ Because limitations in mobility are often the first signs of further functional decline, ${ }^{3,4}$ identifying adults with reduced mobility has been advocated as a key issue in preventing disability in daily life activities. ${ }^{5}$ Thus, an appropriate, simple discriminative tool for identifying people at risk for disability progression in the near future is important, particularly in routine clinical care.

Mobility in patients with COPD can be assessed either through self-report or through performance measures. ${ }^{6}$ Selfreport measures typically assess the subject's perceived difficulties associated with a variety of activities. Performancebased measures rely on a rater's assessment of a subject's performance in a specific, standardized action, measured in a controlled environment. Self-report and performance measures have been used in many studies involving patients with COPD. ${ }^{3,7-10}$

The Short Physical Performance Battery (SPPB) is the most commonly used performance-based measure for patients with COPD. It has gained popularity in the past years, because it is a standardized objective tool, it is rapid and simple to conduct, and it is less influenced by cultural and educational backgrounds, compared with self-report measures..$^{7-10}$ The SPPB represents the sum of results from three component tests of functional relevance: standing balance, 4-meter gait speed (4MGS), and five-repetition sit-tostand motion (5STS). Each component is scored based on a subscale, and the three subscores are added to obtain a summary score. ${ }^{11}$ Because lower extremity strength is important for satisfactory completion of these mobility activities, the SPPB has also been cited as a measure of lower-extremity function. $^{7}$

It has been suggested that the SPPB summary score is a valid measure for identifying patients with quadriceps strength impairments. ${ }^{9}$ However, to our knowledge, it remains to be determined whether it has clinical utility for identifying mobility limitations in patients with COPD. Recently, several studies have shown that two components of the SPPB (4MGS and 5STS) are affected in COPD, but they did not explore the clinical validity of using these measures to screen for mobility limitations. ${ }^{12,13}$

The primary aim of this paper was to determine the clinical validity of the SPPB summary score for identifying mobility limitations in patients with COPD. A secondary aim was to assess whether any component of the SPPB might be equivalent to the full test battery in terms of clinical validity and value for identifying limitations in mobility. Our specific objectives were: 1) determine whether the SPPB summary score could be used to identify patients with mobility limitations; 2) determine which of the three SPPB components could best identify patients with mobility limitations; and 3) compare the validity of the SPPB summary score with that of the best discriminative component in the test battery.

\section{Methods \\ Participants}

Patients with stable COPD were prospectively recruited from an outpatient pulmonary service at Morales Meseguer Hospital, Murcia. Spain. The diagnosis of COPD and the stage of the severity of the disease were based on the Global Initiative for Chronic Obstructive Lung Disease (GOLD) guidelines. ${ }^{14}$ COPD patients with a forced expiratory volume in 1 second/forced vital capacity ratio of $<70 \%$ of the predicted value and age 40-80 years were qualified to participate. Patients were excluded when they had an unstable cardiac condition within 4 months prior to the study, cognitive deterioration, or a limitation in walking. During a 1-year period, a consecutive sample of eligible patients was identified from patient health examinations. A pulmonary physician assessed the eligibility criteria for recruitment.

The Institutional Review Board of the Morales Meseguer Hospital approved the study protocol, and prior informed consent was obtained from all study participants. The study sample included 137 patients.

\section{Procedure}

Patients were approached during visits for monitoring the control of disease. Demographic and clinical information about age, sex, dyspnea, educational achievement, cigarette smoking history, pulmonary function, and GOLD stage was acquired from electronic patient files, interviews, and clinical assessments. Participants were asked to perform the SPPB and strength tests. Knee extension strength was measured first, and then, handgrip strength and SPPB were measured. Finally, self-reported mobility activities were surveyed with a questionnaire, as in previous studies. Two trained clinicians carried out all assessments.

\section{Measures}

\section{Short Physical Performance Battery}

Participants performed the SPPB according to the National Institute on Aging protocol. ${ }^{15}$ The tests were performed in 
the following sequence: a) standing balance tests, b) 4MGS test, and c) 5STS test. The standing balance portion requires participants to maintain, for 10 seconds each, stances with their feet placed side by side, semi-tandem, and in tandem. The scores ranged from 0 to 4 (maximum performance). The 4MGS measured the time needed to walk $4 \mathrm{~m}$ at a typical pace. The 5STS required participants to rise from a chair with their arms across their chest, five times. Categorical scores (range: 0-4) for both the 4MGS and the 5STS tests were based on timed quartiles established previously in a large population. ${ }^{15}$ Individuals who were unable to complete either the 4MGS walking task or the 5STS test received a score of 0 . The sum of the three components comprised the final SPPB score, with a possible range from 0 to 12 . A score of 12 indicated the highest degree of lower extremity functioning. ${ }^{15,16}$ Standardized instructions were given for each of the three SPPB components, and we used standardized equipment for all patients. For example, all sit-to-stand maneuvers were performed from a chair, without armrests, with a seat positioned at a height of $43 \mathrm{~cm}$ and with a depth of $47.5 \mathrm{~cm} .^{11}$

\section{Self-report mobility questionnaire}

Self-reported limitations in mobility were measured with a previously validated self-report questionnaire, described by Eisner et $\mathrm{al}^{7}$ for patients with COPD. ${ }^{6,8}$ The scale comprised ten items, and the patient assessed the degree of difficulty in performing the activity, based on the following response options: none, a little, some, a lot, do not do it, on doctor's orders, unable to do it, and never do that activity. These ten items covered several domains of mobility defined by the ICF, including: five items related to "changing and maintaining body position" (stooping, crouching, or kneeling; standing in place for 15 minutes or longer; getting up from a stooping, crouching, or kneeling position; sitting for long periods; and standing up after sitting in a chair); three items corresponded to "carrying and moving objects" (pushing objects like a living room chair; lifting or carrying light items, under $10 \mathrm{lb}$ or $4.54 \mathrm{~kg}$; and lifting or carrying heavy items over $10 \mathrm{lb}$ ); and two items concerned "walking and moving" (walking alone up and down a flight of stairs; walking two to three neighborhood blocks). This questionnaire was used to classify subjects with or without mobility limitations. Subjects were classified as having a mobility limitation when they indicated that they had "some" or more difficulty with one or more items, or when they were told by a doctor not to do something. Those subjects who indicated "never do that activity" were excluded for the analysis.

\section{Muscle strength measures}

Knee extension and handgrip strength were measured on the dominant side. Muscle strength of knee extensors was measured with a calibrated dynamometer. The testing positions used for assessing muscle strength were based on previous descriptions, ${ }^{17,18}$ and on the guidelines provided in the dynamometer manufacturer's manual. For the quadriceps strength assessment, participants remained seated on a raised plinth, and the assessor placed the participant's knee in a flexed position at 70 degrees. Grip strength was measured with a hydraulic hand dynamometer. In a seated position, each subject kept his or her arm at the side, with the shoulder adducted and neutrally rotated, the elbow flexed at 90 degrees, and the forearm in a neutral position between supination and pronation. The examiner stabilized the elbow, and the subject was asked to squeeze the dynamometer, exerting a maximum grip. All measurements of knee and handgrip strength were repeated two times for each participant, and the individual average was calculated and used for the analysis. ${ }^{19}$

\section{Statistical analysis}

Descriptive statistics were used to describe sociodemographic and clinical characteristics of the participants. To assess the ability of the SPPB summary score to discriminate patients with mobility limitation from those without, we used several measures of diagnostic accuracy. We developed receiver operating characteristic (ROC) curves and calculated the area under the curve (AUC). According to Hosmer and Lemeshow, ${ }^{20}$ an AUC $>0.7$ was used as the criterion of good discrimination. In addition, the ROC analysis allowed us to define the best cutoff score for discriminating some mobility limitations, based on the sensitivity ( $\mathrm{Se}$ ) and specificity (Sp) values associated with the scores of the various SPPB component tests. We chose the value for which $\mathrm{Se}+\mathrm{Sp}-1$ was maximized as the best cutoff point because this point provides the best balance between the proportions of patients correctly identified as having mobility limitation (Se) against the proportions of patients incorrectly identified as having mobility limitation (1- Sp).

Furthermore, from these values of Se and $\mathrm{Sp}$, we calculated positive and negative likelihood ratios to determine the best cutoff scores.

We designed several constructs of validation strategies (known-groups, convergent validity, and divergent validity), where we hypothesized various associations and evaluated the extent to which our data supported the hypotheses. Known-group validity was examined by defining two subgroups for each of the ten items on the self-report mobility 
questionnaire. One subgroup comprised participants who reported no difficulty and the other subgroup comprised those who reported "some" or more difficulty or were told by a doctor not to do the activity. For each item, we hypothesized that mean SPPB score and the mean score of the best discriminative component would be lower in patients who reported difficulty than in those who reported no difficulty. A Mann-Whitney $U$ statistic was used to test for differences in mean scores between the two subgroups. Also, the mean SPPB scores and the mean score of its best discriminative component were compared across groups of patients in different GOLD stages with a Mann-Whitney $U$ statistic. Our hypothesis was that patients classified in GOLD stages with lower symptom levels (A and C) would score higher in the SPPB than those in stages with higher symptom levels (B and D).

Convergent validity was tested by calculating Spearman's correlation coefficients to determine whether the SPPB and its best discriminative component were related to quadriceps muscle strength. We hypothesized that the SPPB score would correlate positively with quadriceps muscle strength, but only to a moderate degree $(0.4<r<0.7)$, because muscle strength belongs to a body function domain on the ICF, as opposed to the mobility domain. Furthermore, for divergent validity, we calculated correlations between SPPB and muscle handgrip strength. We hypothesized that the SPPB would correlate positively, but weakly $(0.2<r<0.4),{ }^{21}$ to handgrip strength, because the SPPB focuses on lower-extremity activities.

Sample size calculation was based on the ability to detect an AUC of 0.71 for SPPB summary score. A total sample size of 95 patients was required assuming an AUC of 0.5 as null hypothesis (meaning no discriminating power), $\alpha=0.05$, $\beta=0.20$, and an expected ratio of 5 between sample size of the group with mobility limitation versus the group without.

All analyses were performed with the SPSS statistical software program (SPSS Version 19.0; IBM SPSS Inc., Chicago, IL, USA). $P<0.05$ was considered statistically significant.

\section{Results}

\section{Participant characteristics}

Of the 147 patients with COPD initially enrolled, ten did not meet study inclusion criteria. Therefore, 137 patients were finally included. The average age of the sample was 66.9 years (range: $46-80$ years); most were male $(87.6 \%)$, and most had less than a high school education $(72.8 \%$; Table 1). Regarding clinical characteristics, the majority of participants were GOLD stage D.
Table I Baseline characteristics

\begin{tabular}{|c|c|}
\hline Characteristics & n (\%) or mean (SD) \\
\hline \multicolumn{2}{|l|}{ Demographic } \\
\hline Age, mean (SD) & $66.9(8.3)$ \\
\hline Male, $n(\%)$ & $120(87.6 \%)$ \\
\hline \multicolumn{2}{|l|}{ Educational achievement, n (\%) } \\
\hline Primary studies & 99 (72.8\%) \\
\hline High school & $24(17.6 \%)$ \\
\hline University & $13(9.6 \%)$ \\
\hline \multicolumn{2}{|l|}{ Clinical characteristics } \\
\hline $\mathrm{FEV}_{1}(\mathrm{~L})$, mean $(\mathrm{SD})$ & $1.29(0.46)$ \\
\hline $\mathrm{FEV}_{\text {, }}(\%$ predicted), mean (SD) & $50.21(16.5)$ \\
\hline $\mathrm{FVC}(\mathrm{L})$, mean $(\mathrm{SD})$ & $2.22(0.68)$ \\
\hline FVC (\% predicted), mean (SD) & $66.7(18.74)$ \\
\hline \multicolumn{2}{|l|}{ Smoking history, n (\%) } \\
\hline Current smoker & $4 \mathrm{I}(29.9 \%)$ \\
\hline Former smoker & 96 (70.1\%) \\
\hline Never smoked & $0(0 \%)$ \\
\hline \multicolumn{2}{|l|}{ GOLD group, n (\%) } \\
\hline A & $24(I 7.5 \%)$ \\
\hline B & $22(16.1 \%)$ \\
\hline $\mathrm{C}$ & $12(8.8 \%)$ \\
\hline $\mathrm{D}$ & $79(57.7 \%)$ \\
\hline \multicolumn{2}{|l|}{ CAT category, n (\%) } \\
\hline $0-10$ & $37(27 \%)$ \\
\hline$\geq 10$ & $100(73 \%)$ \\
\hline \multicolumn{2}{|l|}{ Exacerbations in last year, $\mathbf{n}(\%)$} \\
\hline $0-1$ & $58(42.3 \%)$ \\
\hline$\geq 2$ & 79 (57.7\%) \\
\hline mMRC score, median (interquartile range) & $1.0(1.0)$ \\
\hline \multicolumn{2}{|l|}{ Airflow obstruction, n (\%) } \\
\hline Slight & $5(3.6 \%)$ \\
\hline Moderate & 71 (5I.8\%) \\
\hline Severe & 50 (36.5\%) \\
\hline Very severe & II (8\%) \\
\hline
\end{tabular}

Abbreviations: SD, standard deviation; $\mathrm{FEV}_{1}$, forced expiratory volume in I second; FVC, forced vital capacity; GOLD, Global Initiative for Chronic Obstructive Lung Disease; CAT, COPD Assessment Test; mMRC, modified Medical Research Council.

A total of 115 participants had self-reported mobility limitations in one or more of the ten items on the SPPB. A mean of 3.9 (standard deviation $=2.3$ ) mobility activities were rated as difficult.

\section{Measures of diagnostic accuracy}

The ROC curves and AUCs of the full test battery and the three test components are shown in Figure $1 \mathrm{~A}$ and $\mathrm{B}$, respectively. The AUC for the summary SPPB score was $0.75(95 \%$ confidence interval [CI]: 0.66-0.85). The AUC values for the 5STS test, the 4MGS test, and the balance tests were 0.75 (95\% CI: 0.65-0.84), 0.60 (95\% CI: 0.47-0.71), and 0.60 (95\% CI: 0.48-0.71), respectively. Only the 5STS and full battery tests showed good discrimination (AUC $>0.7$ ). Analysis with continuous data did not substantially change these results (5STS $\mathrm{AUC}=0.74$, 4MGS $\mathrm{AUC}=0.66$ ). 
A

ROC curve for SPPB summary score

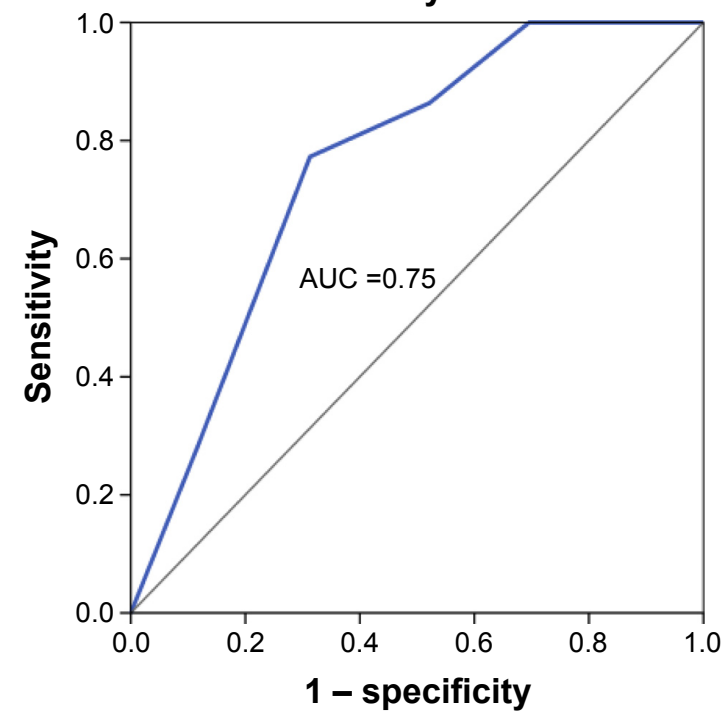

B ROC curves for SPPB components scores

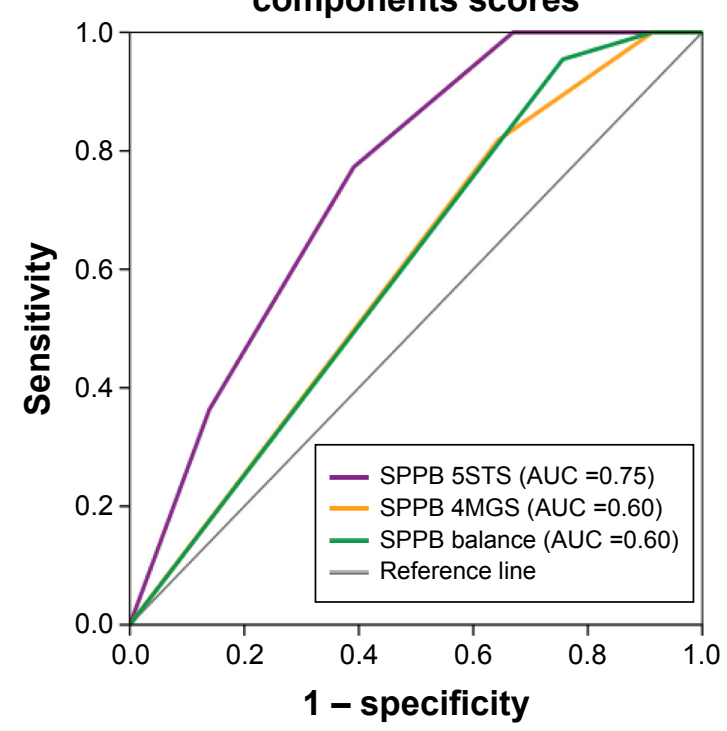

Figure I ROC curves for SPPB scores.

Notes: Lines show the specificity and sensitivity for the SPPB $(\mathbf{A})$ and for the different test components (B) in determining functional limitations in mobility. Abbreviations: ROC, receiver operating characteristic; SPPB, Short Physical Performance Battery; 5STS, five-repetition sit-to-stand motion; 4MGS, 4-meter gait speed; AUC, area under the curve.

For the SPPB summary score, a cutoff score of 10 provided the most accurate discrimination of mobility limitations. With this cutoff score, we calculated Se and Sp values of 0.77 and 0.70 , respectively. Based on the cutoff score and the corresponding Se and Sp values, we calculated positive and negative likelihood ratios of 2.56 and 0.32 , respectively, for mobility limitation.

\section{Construct validity}

Table 2 shows the means of the SPPB summary scores and 5STS tests for the subgroup with some difficulty and the subgroup with no difficulty for each of the ten mobility activities on the self-report questionnaire. As expected, the mean of all SPPB scores differed significantly between subgroups, except for the item "sitting for 1 hour". The differences between groups in the mean scores of the full test battery ranged from 1.71 to 1.13 points for the nine items, with lower scores for the group that indicated some difficulty. The differences between groups in the mean scores of the 5STS test ranged from 0.98 to 0.59 points; again, with lower scores for the group that indicated some difficulty. The validity of this known-groups construct was further supported by the fact that participants in GOLD stages B and D had lower mean scores than participants in GOLD stages A and C. The mean SPPB summary scores were 9.18 and 10.64 , respectively $(P=0.001)$, and the mean 5STS test scores were 2.02 versus 3.03 , respectively $(P=0.001)$.
The correlation coefficients for the mean SPPB score or mean 5STS score and the muscle strength measures are shown in Table 3. As hypothesized, moderate correlations were observed between both SPPB scores and the proportion of activities with difficulty and knee extension muscle strength. In addition, as hypothesized, the correlations between the mobility tests and the handgrip muscle strength did not achieve the recommended level of 0.4 , which indicated a divergent validity.

\section{Discussion}

Our findings provided evidence for the validity of the full performance test battery and the 5STS test as clinical tools for assessing mobility limitations in patients with COPD. Moreover, both measures were significantly related to the capacity to perform physical activities important in daily living, such as changing and maintaining body position; carrying, moving, and handling objects; or walking and ambulation.

In the present study, we tested whether any one of the three SPPB components (5STS, 4MGS, and standing balance) were suitable for identifying patients with mobility limitations. We found that the 5STS test had the largest AUC value on an ROC analysis. To our knowledge, this was the first study to assess whether one of the three components of the SPPB might provide value equivalent to the SPPB for assessing patients with COPD. ${ }^{9}$ In our opinion, the 5STS 
Table 2 Mean SPPB scores according to self-reported functional limitations

\begin{tabular}{|c|c|c|c|}
\hline Domains of mobility activities & No difficulty $\left(n_{1}\right)$ & Difficulty $\left(\mathrm{n}_{2}\right)$ & $P$-value (t-test) \\
\hline \multicolumn{4}{|c|}{ Changing and maintaining body position } \\
\hline \multicolumn{4}{|c|}{ Stooping, kneeling $\left(n_{1}=50 ; n_{2}=86\right)$} \\
\hline SPPB summary score & 10.24 & 9.16 & 0.001 \\
\hline SPPB 5STS & 2.68 & 2.05 & 0.002 \\
\hline \multicolumn{4}{|l|}{ Standing $>15$ minutes $\left(n_{1}=86 ; n_{2}=5 I\right)$} \\
\hline SPPB summary score & 10.16 & 8.55 & $<0.001$ \\
\hline SPPB 5STS & 2.57 & 1.80 & $<0.001$ \\
\hline \multicolumn{4}{|c|}{ Getting up from a kneeling position $\left(n_{1}=43 ; n_{2}=93\right)$} \\
\hline SPPB summary score & 10.51 & 9.12 & $<0.00$ I \\
\hline SPPB 5STS & 2.95 & 1.97 & $<0.00$ I \\
\hline \multicolumn{4}{|l|}{ Sitting I hour $\left(n_{1}=100 ; n_{2}=37\right)$} \\
\hline SPPB summary score & 9.54 & 9.62 & 0.873 \\
\hline SPPB 5STS & 2.29 & 2.27 & 0.944 \\
\hline \multicolumn{4}{|c|}{ Standing from a seated position $\left(n_{1}=109 ; n_{2}=27\right)$} \\
\hline SPPB summary score & 9.90 & 8.30 & 0.001 \\
\hline SPPB 5STS & 2.48 & 1.56 & 0.001 \\
\hline \multicolumn{4}{|c|}{ Carrying, moving, and handling objects } \\
\hline \multicolumn{4}{|c|}{ Pushing objects $\left(n_{1}=108 ; n_{2}=28\right)$} \\
\hline SPPB summary score & 9.82 & 8.57 & 0.001 \\
\hline SPPB 5STS & 2.42 & 1.79 & 0.01 \\
\hline \multicolumn{4}{|l|}{ Lifting light objects $\left(n_{1}=110 ; n_{2}=27\right)$} \\
\hline SPPB summary score & 9.82 & 8.52 & 0.006 \\
\hline SPPB 5STS & 2.40 & 1.81 & 0.032 \\
\hline \multicolumn{4}{|l|}{ Lifting heavy objects $\left(n_{1}=74 ; n_{2}=62\right)$} \\
\hline SPPB summary score & 10.08 & 8.95 & $<0.00$ I \\
\hline SPPB 5STS & 2.58 & 1.94 & 0.002 \\
\hline \multicolumn{4}{|l|}{ Walking and moving } \\
\hline \multicolumn{4}{|l|}{ Walking up stairs $\left(n_{1}=105 ; n_{2}=32\right)$} \\
\hline SPPB summary score & 9.96 & 8.25 & $<0.001$ \\
\hline SPPB 5STS & 2.50 & 1.59 & $<0.001$ \\
\hline \multicolumn{4}{|c|}{ Walking in the neighborhood $\left(n_{1}=87 ; n_{2}=48\right)$} \\
\hline SPPB summary score & 10.16 & 8.54 & $<0.001$ \\
\hline SPPB 5STS & 2.63 & 1.69 & $<0.001$ \\
\hline
\end{tabular}

Abbreviations: SPPB, Short Physical Performance Battery; 5STS, five-repetition sit-to-stand motion.

test had the highest value for assessing mobility limitations, because standing up from a sitting position is a very common, essential activity that reflects the potential for other vital daily life activities, such as walking or carrying a bag. ${ }^{22}$

The results from analyses of the 4MGS and balance tests showed that these were not relevant factors in the discrimination of mobility limitations. Our 4MGS result was inconsistent with previous findings in a general geriatric population. ${ }^{23}$ Moreover, in previous studies, the 4MGS test was highly

Table 3 Evidence for convergent/divergent validity based on correlation coefficients between scores on two tests

\begin{tabular}{lll}
\hline Measures & Quadriceps strength & Handgrip strength \\
\hline SPPB summary score & 0.49 & 0.28 \\
SPPB 5STS & 0.41 & 0.18 \\
\hline
\end{tabular}

Abbreviations: SPPB, Short Physical Performance Battery; 5STS, five-repetition sit-to-stand motion. related to numerous physiological and pulmonary functions in patients with COPD, such as dyspnea and exercise capacity, measured with the 6-minute walk test or steps per day. ${ }^{24}$ It is likely that the small distance involved in the 4MGS test could have explained our finding that it lacked the ability to identify functional limitations in patients with COPD.

The construct validity of both the full test battery and the 5STS test was supported by differences in scores across groups of patients who reported different levels of difficulty in a variety of mobility activities. We analyzed patient's difficulty with activities that pertained to different mobility domains in the ICF, similar to another validity study performed previously in a general geriatric population. ${ }^{25}$ Our findings were consistent with the findings in that study. The results showed that only one activity was not identified with either of the two tests: sitting for long periods. A possible 
explanation for this result might be that this activity was the most sedentary in the entire questionnaire. ${ }^{26}$

The construct validity of both tests was also supported by differences in scores across groups in different GOLD stages. We compared groups in GOLD stages $\mathrm{A}$ and $\mathrm{C}$ groups with groups in GOLD stages B and D. We found that health status (based on dyspnea, COPD Assessment Test scores, airflow limitation, and risk of exacerbations) was correlated to the SPPB summary score. ${ }^{9}$ Our study also suggested that health status could be a specific determinant for performance on the 5STS test. However, more research is needed to investigate this issue.

As hypothesized, we found that the SPPB scores were moderately correlated with the quadriceps strength measurement and only slightly correlated with the handgrip strength measurement. It is well known that bodily functions, such as muscle strength, are related to functional activities, ${ }^{1,2,19,27}$ partly because a certain amount of strength is necessary for the satisfactory completion of most functional activities. From this perspective, and because the activities in the SPPB rely mostly on lower-extremity strength, we expected the SPPB scores to be more highly correlated with quadriceps strength than with handgrip muscle strength.

\section{Limitations}

Despite our findings that the SPPB generally showed good clinical validity, several limitations should be noted. First, in this study, we measured limitations in mobility with a self-report questionnaire to test the clinical validity of the SPPB. It is well known that the mobility functions addressed in the SPPB do not provide nearly as broad a view as those addressed in the self-report used in this study. Thus, direct comparisons might be challenging. However, both modes of measurement are considered measures of the mobility domain, and both are advocated as outcomes of this domain. ${ }^{7,8,10}$ Second, this study was restricted to a single region of Spain; therefore, the discriminative ability of the SPPB may not be generalizable to different settings. Third, we used only cross-sectional strategies to test construct validity. Future studies should consider these associations prospectively, and a broader range of locations should be included. Finally, we defined mobility limitation as having some or higher level of difficulty in one or more mobility activities. Thus, the group of patients with mobility limitation had a wide range of mobility. Nevertheless, we used that definition for mobility limitation because our interest was to evaluate SPPB as a screening tool to detect cases even with low levels of limitation.

\section{Implications for practice and research}

The potential applications of the findings from the present study are numerous. Thus, based on our finding that the SPPB cutoff value was 10 , patients with lower values can be considered to have one or more mobility limitations. Moreover, this cutoff value may also be used to identify patients with COPD that have impaired quadriceps muscle strength, as described by Patel et al. ${ }^{9}$ Nevertheless, the cutoff value is relevant only as a screening tool. If the objective of the clinical trial is to identify the specific area of mobility limitation or specific muscle strength, a self-reported mobility questionnaire or a dynamometer should be used.

This study demonstrated that the stance assessment alone was nearly as accurate as the full test battery for discriminating a mobility limitation. This is a valuable finding, because it supports the use of this test when time is limited in clinical settings. However, we cannot confidently recommend abandoning the full performance test battery. Certainly, assessments of gait speed and balance can be of value in understanding the patient's experience of this type of disability. ${ }^{28}$ Moreover, gait speed alone is a good indicator of other issues, such as health care utilization ${ }^{29}$ and survival. ${ }^{30}$

The greatest value of our present findings on performancebased measures is that they provide a strategy for preventing future disabilities by identifying people who have mobility limitations with a simple screening test. Consequent to the screening results, it may be necessary to complement medical management with comprehensive rehabilitation strategies aimed at the diverse mobility limitations of patients with COPD. This dual strategy may prevent disability and restore normal function.

Although the results of the present study provide evidence that the SPPB is clinically valid for identifying mobility limitations, it is important to recognize the scope of the present study and consider future research directions. For example, more studies could assess whether each of three SPPB components (balance, 4MGS, and 5STS) are equivalent in the value they add to other measures of COPD manifestations, such as aerobic capacity measured with the 6-minute walk test. Furthermore, future research could explore the value of the SPPB and 5STS tests as indicators of health care utilization and survival in patients with COPD.

\section{Conclusion}

This study provided evidence for validity of the SPPB summary score and the 5STS test for assessing mobility in patients with COPD. Our results showed that these tests had 
equivalent potential as screening tools for identifying patients with mobility limitations.

\section{Acknowledgments}

The authors wish to thank the patients and personnel of the hospital unit for their cooperation during the course of this study. The study was supported by medical manager of AstraZeneca Pharmaceutical Spain, S.A. and Maite Pérez.

\section{Disclosure}

The authors report no conflicts of interest in this work.

\section{References}

1. Katz PP, Gregorich S, Eisner MD, et al. Disability in valued life activities among individuals with COPD and other respiratory conditions. J Cardiopulm Rehabil Prev. 2010;30(2):126-136.

2. World Health Organization. International Classification of Functioning, Disability, and Health. Geneva: World Health Organization; 2002.

3. Eisner MD, Iribarren C, Blanc PD, et al. Development of disability in chronic obstructive pulmonary disease: beyond lung function. Thorax. 2011;66(2):108-114.

4. Guralnik JM, Ferrucci L, Simonsick EM, Salive ME, Wallace RB. Lower-extremity function in persons over the age of 70 years as a predictor of subsequent disability. N Engl J Med. 1995;332(9):556-561.

5. Studenski S, Perera S, Wallace D, et al. Physical performance measures in the clinical setting. J Am Geriatr Soc. 2003;51(3):314-322.

6. Gill TM. Assessment of function and disability in longitudinal studies. J Am Geriatr Soc. 2010;58(Suppl 2):S308-S312.

7. Eisner MD, Iribarren C, Yelin EH, et al. Pulmonary function and the risk of functional limitation in chronic obstructive pulmonary disease. Am J Epidemiol. 2008;167(9):1090-1101.

8. Eisner MD, Blanc PD, Yelin EH, et al. COPD as a systemic disease: impact on physical functional limitations. Am J Med. 2008;121(9):789-796.

9. Patel MS, Mohan D, Andersson YM, et al. Phenotypic characteristics associated with reduced Short Physical Performance Battery score in COPD. Chest. 2014;145:1016-1024.

10. Eisner MD, Blanc PD, Sidney S, et al. Body composition and functional limitation in COPD. Respir Res. 2007;8:7.

11. Guralnik JM. Assessing physical performance in the older patient [CDROM]. Bethesda, MD: National Institutes of Aging. Available from: http://www.grc.nia.nih.gov/branches/leps/sppb/. Updated June 5, 2013. Accessed January 10, 2010.

12. Jones SE, Kon SS, Canavan JL, et al. The five-repetition sit-to-stand test as a functional outcome measure in COPD. Thorax. 2013;68(11): 1015-1020.

13. Kon SS, Patel MS, Canavan JL, et al. Reliability and validity of the 4-metre gait speed in COPD. Eur Respir J. 2013;42(2):333-340.
14. Global Strategy for the Diagnosis, Management and Prevention of COPD, Global Initiative for Chronic Obstructive Lung Disease (GOLD) 2015. Available from: http://www.goldcopd.org/. Accessed September 25, 2015.

15. Guralnik JM, Simonsick EM, Ferrucci L, et al. A short physical performance battery assessing lower extremity function: association with self-reported disability and prediction of mortality and nursing home admission. J Gerontol. 1994;49(2):M85-M94.

16. American Thoracic Society/European Respiratory Society. ATS/ERS Joint statement on respiratory muscle testing. Am J Respir Crit Care Med. 2002;166(4):518-624.

17. Taylor N, Dodd K, Graham H. Test retest reliability of hand-held dynamometric strength-testing for young people with cerebral palsy. Arch Phys Med Rehabil. 2004;85(1):77-80.

18. Damiano D, Abel M. Functional outcomes of strength training in spastic cerebral palsy. Arch Phys Med Rehabil. 1998;79(2):119-125.

19. Mathiowetz V, Weber K, Volland G, Kashman N. Reliability and validity of grip and pinch strength evaluations. J Hand Surg Am. 1984;9(2): 222-226.

20. Hosmer DW, Lemeshow S. Applied Logistic Regression. 2nd ed. Hoboken, NJ: John Wiley \& Sons; 2000.

21. Correlation explained (Time Web). Available from: http://www. sunshineprofits.com/gold-silver/dictionary/correlation-coefficient/. Accessed November 17, 2015.

22. Ozalevli S, Ozdena AO, Itil O, Akkoclub A. Comparison of the sitto-stand test with 6 min walk test in patients with chronic obstructive pulmonary disease. Respir Med. 2007;101(2):286-293.

23. Guralnik JM, Ferrucci L, Pieper CF, et al. Lower extremity function and subsequent disability: consistency across studies, predictive models, and value of gait speed alone compared with the short physical performance battery. J Gerontol A Biol Sci Med Sci. 2000;55(4):221-231.

24. Karpman C, Benzo RP. Gait speed as a measure of functional status in COPD patients. Int J Chron Obstruct Pulmon Dis. 2014;9:1315-1320.

25. Freire AN, Gurra RO, Alvarado B, et al. Validity and reliability of the short physical performance battery in two diverse older adult populations in Quebec and Brazil. J Aging Health. 2012;24(5):863-878.

26. Sternfeld B, Ngo L, Satariano WA, Tagern IB. Associations of body composition with physical performance and self-reported functional limitation in elderly men and women. Am J Epidemiol. 2002;156(2): $110-121$.

27. van de Ven-Stevens LA, GraffMJ, Peters MA, van der Linde H, Geurts AC. Construct validity of the Canadian occupational performance measure in participants with tendon injury and Dupuytren disease. Phys Ther. 2015;95(5):750-757.

28. Puthoff ML. Outcome measures in cardiopulmonary physical therapy: short physical performance battery. Cardiopulm Phys Ther J. 2008; 19(1):17-22.

29. Ostir GV, Berges I, Kuo YF, Goodwin JS, Ottenbacher KJ, Guralnik JM. Assessing gait speed in acutely ill older patients admitted to an acute care for elders hospital unit. Arch Intern Med. 2012;172(4):353-358.

30. Studenski S, Perera S, Patel K, et al. Gait speed and survival in older adults. JAMA. 2011;305(1):50-58.
International Journal of COPD

\section{Publish your work in this journal}

The International Journal of COPD is an international, peer-reviewed journal of therapeutics and pharmacology focusing on concise rapid reporting of clinical studies and reviews in COPD. Special focus is given to the pathophysiological processes underlying the disease, intervention programs, patient focused education, and self management protocols.

\section{Dovepress}

This journal is indexed on PubMed Central, MedLine and CAS. The manuscript management system is completely online and includes a very quick and fair peer-review system, which is all easy to use. Visit http://www.dovepress.com/testimonials.php to read real quotes from published authors. 